\title{
Peculiar behavior of solar polar fields during solar cycles 21-23: Correlation with meridional flow speed
}

\author{
Susanta Kumar Bisoi and P. Janardhan
}

Physical Research Laboratory, Astronomy \& Astrophysics Division, Ahmedabad 380 009, India email: susanta@prl.res.in

\begin{abstract}
We have examined solar polar fields during the solar cycles 21-23 using both ground and space based synoptic magnetograms. It has been shown that the unsigned polar fields in the latitude range $\left(78^{\circ}-90^{\circ}\right)$ show a steady decline starting $\sim 1995-1996$ till the maximum of cycle 23. We also find that the long term changes in the unsigned polar fields at higher latitudes are well correlated with changes in the surface meridional flow speed that have been reported for the cycle 23 .
\end{abstract}

Keywords. Sun: magnetic fields, Solar cycle, and Meridional flow.

\section{Introduction}

The generation and evolution of solar magnetic fields in a solar cycle invlove three basic processes governed by an internal solar dynamo viz. the generation of toroidal fields from pre-existing poloidal fields ( $\omega$ - effect), the re-generation of poloidal fields by twisting of toroidal flux tubes ( $\alpha$-effect), and magnetic flux transport by meridional circulation. Solar polar fields are formed as the background magnetic flux accumulates polewards due to the cumulative action of surface meridional flows and diffusion. Polar fields reverse around every solar maximum and the Sun's memory of the polar field strength at the solar minimum is a crucial factor in predicting the amplitude of upcoming solar cycles. Hence, we have examined solar polar fields in the high latitude range using the data from the National Solar Observatory, Kitt Peak, (NSO/KP) covering the years 19752009 and data from the MDI instrument onboard the Solar and Heliospheric Observatory (SOHO/MDI) covering the years 1996-2009 (for details refer Janardhan et. al, 2010 and references therein).

\section{Result and Implications}

For the estimation of magnetic fields, all synoptic maps, in the form of $180 \times 360$ arrays, are first longitudinally averaged into a $1^{\circ}$ wide longitude strip. Each of these strips are then averaged in the latitude range, $\left(78^{\circ}-90^{\circ}\right)$ to obtain one magnetic field value for each CR. Figure 1 shows the magnetic field variations obtained for each $\mathrm{CR}$ for the northern (upper panel) and southern (lower panel) solar hemispheres as function of time in years (filled dots for the NSO/KP and triangles for the MDI) for the solar cycles 21,22 and 23 . The missing values in the data are replaced by interpolated values obtained via cubic spline method (stars in grey for the interpolated NSO/KP and the open circles for the interpolated MDI). The solid smoothed curve in both the panels show the behavior of solar polar fields for the last three solar cycles.

A steady decline in the unsigned (absolute value) polar fields has been observed as seen from Fig 1 both in the NSO/KP and the SOHO/MDI data in the northern hemispheres 


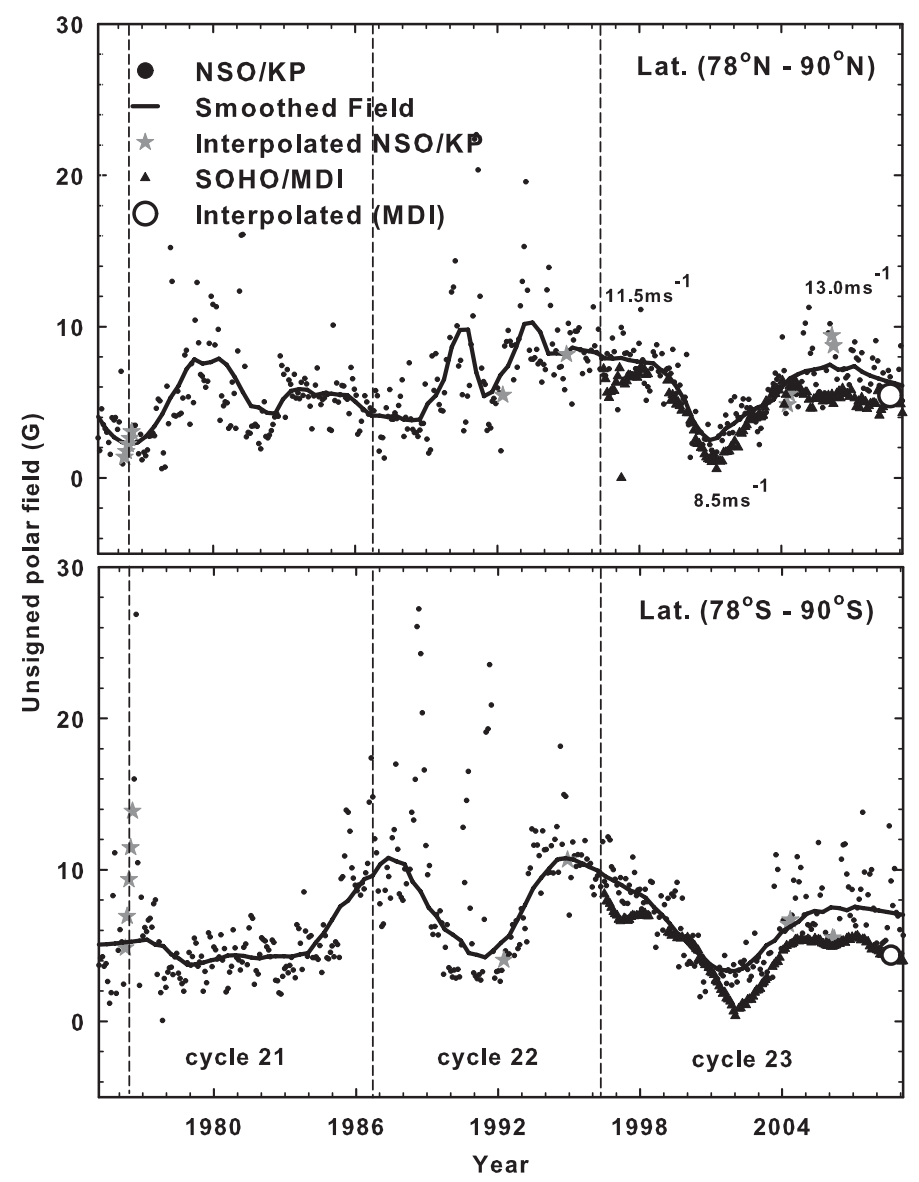

Figure 1. shows the unsigned (absolute) solar polar field variations in the latitude range, $\left(78^{\circ}-90^{\circ}\right)$, for the north and south solar hemispheres as function of years for the cycles 21,22 and 23 for the NSO/Kitt Peak and for the cycle 23 for the SOHO/MDI. The legends (at the top left of the uppper panel) decribe the symbols used in the Figure while the vertical lines demarcate the three cycles 21, 22 and 23. The meridional flow speeds as reported by Hathaway \& Rightmire (2010) are indicated at different phases of the cycle 23.

1994-2001 and in the southern hemispheres 1995-2002 which has continued till maximum of the cycle 23. In addition, compared to the previous solar cycles 22 and 23 , we find very weaker polar fields in the cycle 23 , with the lowest being at the maximum of the cycle 23 and then recovering back and staying almost constant after 2004. Furthermore, the meridional flow speeds reported by Hathaway \& Rightmire (2010) behaved in a similar manner to the polar fields which were high $\left(11.5 \mathrm{~ms}^{-1}\right)$ during 1996-1997, dropped down to $8.5 \mathrm{~ms}^{-1}$ during 2000-2001, increased to $13.5 \mathrm{~ms}^{-1}$ and remained constant after 2004. The high meridional flow speed at the end of the cycle 23 would have caused this weak polar field which eventually lead to the prolonged minimum. Since the solar memory depends on polar field strength the weak polar field in the cycle 23 would be carried to the next cycle 24 thereby causing a weaker solar maximum.

\section{References}

Hathaway, D. H. \& Rightmire, L. 2010, Science, 327, 1350

Janardhan, P., Bisoi, S. K., \& Gosain, S. 2010, Solar Phys., 267, 267 